\title{
The effects of concurrent task interference on category learning: Evidence for multiple category learning systems
}

\author{
ELLIOTT M. WALDRON and F. GREGORY ASHBY \\ University of California, Santa Barbara, California
}

\begin{abstract}
Participants learned simple and complex category structures under typical single-task conditions and when performing a simultaneous numerical Stroop task. In the simple categorization tasks, each set of contrasting categories was separated by a unidimensional explicit rule, whereas the complex tasks required integrating information from three stimulus dimensions and resulted in implicit rules that were difficult to verbalize. The concurrent Stroop task dramatically impaired learning of the simple explicit rules, but did not significantly delay learning of the complex implicit rules. These results support the hypothesis that category learning is mediated by multiple learning systems.
\end{abstract}

There has been much recent interest in the hypothesis that performance in many cognitive tasks is simultaneously mediated by qualitatively distinct processing systems. Arguments for multiple systems have come from such diverse fields as memory (Mishkin, Malamut, \& Bachevalier, 1984; Schacter, 1987; Squire, 1992), reasoning (Sloman, 1996), motor learning (Willingham, Nissen, \& Bullemer, 1989), discrimination learning (Kendler \& Kendler, 1962), and function learning (Hayes \& Broadbent, 1988). In general, these studies have suggested at least two distinct types of learning systems: (1) an explicit, rule-based system tied to language functions and conscious awareness, and (2) an implicit or habit-forming system, which may not have access to conscious awareness.

Within the domain of category learning, there also were early arguments for multiple systems. For example, Brooks (1978) hypothesized that category learning is mediated by separate "deliberate, verbal, analytic control processes and implicit, intuitive, nonanalytic processes" (p. 207). Nevertheless, most quantitative accounts of category learning assume the existence of a single system (e.g., Estes, 1986; Hintzman, 1986; Kruschke, 1992; Medin \& Schaffer, 1978; Nosofsky, 1986). Recently, however, quantitative models that assume multiple category learning systems have been developed (e.g., Ashby, Alfonso-Reese, Turken, \& Waldron, 1998; Erickson \& Kruschke, 1998). For example, Ashby et al. (1998) proposed a formal neuropsychological theory of multiple category learning systems called COVIS (competition between verbal and implicit systems), which assumes

This research was supported by Grant SBR-9514427 from the National Science Foundation. We thank And Turken for his help in designing the experiment described in this article. Correspondence concerning this article should be addressed to F. G. Ashby, Department of Psychology, University of California, Santa Barbara, CA 93106 (e-mail: ashby@psych.ucsb.edu). separate explicit (rule-based) and implicit (procedural learning-based) systems. In response, Nosofsky and Johansen (2000) have argued that single-system (exemplar) models can account for many of the phenomena that have been used to support the notion of multiple systems.

This article presents evidence for the existence of separate category learning systems. Using a concurrent task methodology, we evaluated the ability of observers to learn categories defined by simple or complex rules, while at the same time they performed a numerical analogue of the classic Stroop task (Stroop, 1935). Single-system models of category learning naturally predict that the Stroop task interference observed with the simple category structures should be less than or equal to the interference observed with the complex category structures. Consistent with COVIS, however, the data show strong concurrent task interference with the simple rules and minimal interference with the complex rules. We end with a discussion of the implications that the present results pose for current models of categorization as well as for the more general field of human learning.

\section{Evidence for Multiple Systems}

Empirical evidence for multiple category learning systems comes from several different sources. For example, Ashby, Queller, and Berretty (1999) reported evidence for multiple systems from an unsupervised category learning experiment. It is well known that with supervision (i.e., trial by trial feedback), people can learn complex rules that are difficult or impossible to verbalize (e.g., Ashby \& Maddox, 1992; McKinley \& Nosofsky, 1995; Medin \& Schwanenflugel, 1981). However, in category learning situations where trial by trial feedback is absent, people almost always use unidimensional rules (Ahn \& Medin, 1992; Ashby et al., 1999; Imai \& Garner, 1965; Medin, Wattenmaker, \& Hampson, 1987; Wattenmaker, 1992). Ashby et al. (1999) have shown that without supervision, 
subjects could learn simple unidimensional rules, whereas no learning was seen with more complex two-dimensional rules. ${ }^{1}$ This pattern of results is suggestive of distinct learning systems, where one system can learn in the absence of supervision (i.e., an explicit system) and the other system can only learn under supervised conditions (i.e., an implicit system).

Allen and Brooks (1991) investigated the effects of experimental instructions on classification behavior. Using stimuli that varied on five binary-valued stimulus dimensions, one group of participants was explicitly told the optimal categorization rule before training (the rule group), whereas a second group was told to memorize the category assignments of all stimuli (the memory group). The optimal rule was of the following form: "If the stimulus has at least two of three critical attributes, choose category $A$, otherwise choose category $B$." This rule was complex enough so that participants in the memory group were unlikely to discover it during training. The main result concerned the categorization of a novel stimulus in a subsequent transfer task. This stimulus was designed so that it would be assigned to one category by the optimal rule, even though it was more similar to the exemplars from the other category. Allen and Brooks found that if the participants were given the optimal rule explicitly, they were then more likely to categorize the novel stimulus according to the optimal rule. The participants that were instructed to memorize the stimulus-category relationships, however, were more likely to categorize the novel stimulus as a member of the category with more similar exemplars. In a recent positron emission tomography (PET) study (Smith, Patalano, Jonides, \& Koeppe, 1996; cited in Smith, Patalano, \& Jonides, 1998) done with a similar paradigm, different brain areas were found to be active in the rule and memory conditions. For example, in the rule condition, there was greater activation of frontal areas, whereas the memory condition produced greater activation in more posterior cortical areas. These results are consistent with the notion of multiple category learning systems because they show that task instructions can induce qualitatively different categorization strategies and different patterns of neural activation.

All of these results are consistent with the assumption of multiple category learning systems. Nevertheless, many of them could be accounted for by a single system able to implement decision rules of differing complexity. For example, a single-system model could account for the Ashby et al. (1999) unsupervised results by assuming that simple (e.g., unidimensional) rules can be learned without feedback, but that the learning of complex rules requires supervision. The Allen and Brooks (1991) results could be subject to a similar interpretation. Participants in the memory group might have been using one of the many possible decision rules that would succeed with the training stimuli, whereas the rule group was told which one of these rules to use. Finally, in the Smith et al. (1996) neuroimaging study, there was considerable overlap in patterns of activation between the rule and mem- ory groups, and it is difficult to rule out the possibility that the differences that were seen occurred simply because of the extra learning processes required of the memory group.

\section{Goals of the Present Study}

Our goal in the present study was to provide a stronger test of the multiple-systems hypothesis. We examined the ability of participants to learn explicit (i.e., unidimensional) and implicit (i.e., complex three-dimensional) categorization rules under normal conditions; at the same time, they were performing a secondary task that was designed to interfere with explicit category learning, but not with implicit learning.

The categorization stimuli were colored geometric figures presented on a colored background. The stimuli varied on four binary-valued dimensions: background color (blue or yellow), embedded symbol color (red or green), symbol numerosity (one or two), and symbol shape (square or circle). This yielded a total of 16 possible stimuli. For the explicit category structures, the rule separating the categories was always a unidimensional rule of the following form: "If the background color is blue then choose category $A$, if background color is yellow then choose category $B$." Four different category structures were created by varying which stimulus dimension was critical. We refer to these rules as explicit, because they are easy to describe verbally and because, at the end of training, all participants were able to describe these rules accurately.

We constructed a set of implicit category structures in the following manner. First, one level of each stimulus dimension was arbitrarily assigned the numerical value of -1 , and the other level was assigned a value of +1 (e.g., a background color of blue $=-1$, and a background color of yellow = +1). Next, one of the four stimulus dimensions was selected to be irrelevant. Call the three remaining (relevant) dimensions X, Y, and Z. Finally, stimuli were assigned to categories according to the following rule:

$$
\begin{aligned}
& \text { If value }(\mathrm{X})+\operatorname{value}(\mathrm{Y})+\operatorname{value}(\mathrm{Z})>0, \\
& \quad \text { assign to category } A \text {, } \\
& \text { otherwise assign to category } B \text {. }
\end{aligned}
$$

Four such category structures were created by varying which dimension was irrelevant. We call these rules implicit, because they are extremely difficult to describe verbally. When asked to describe the rule that they used with these implicit structures, none of the participants were able to do so, even though they all learned to categorize the stimuli successfully. For a more complete discussion of explicit and implicit categorization rules, see Ashby et al. (1998).

These explicit and implicit rules are similar to the Type I and Type IV (respectively) rules initially studied by Shepard, Hovland, and Jenkins (1961). ${ }^{2}$ Shepard et al. also studied four other rule types and found that the relative difficulty of the different rules was largely determined by the number of dimensions relevant to the categorization decision - that is, the more the relevant dimensions, 
the more difficult the categorization problem (see Nosofsky, Gluck, Palmeri, McKinley, \& Glauthier, 1994, for a recent replication). It has been suggested that this result occurs because it takes "more cognitive effort or capacity to consider more dimensions" (Kruschke, 1992, p. 26). Thus, empirical evidence suggests that the explicit rules should be easier to learn than the implicit.

How should the addition of a concurrent task affect the learning of the explicit and implicit rules? A concurrent secondary task is commonly thought to reduce processing resources available for a primary task (see, e.g., Pashler, 1994). Accordingly, the learning of any new category structures should be more difficult in the presence of a concurrent task. If more effort or capacity is required in order to learn more complex rules and there is only a single system (or processing resource) that operates on all rules, then the degree of concurrent task interference for the more complex rules should always be greater than or equal to the interference for the less complex rules. ${ }^{3}$ However, if the different rule types are learned by separate systems, then the pattern of concurrent task interference between the rule types should only reflect the relative interference of the concurrent task on each component system. Thus, given separate systems, it is possible that the concurrent task could cause less interference with the complex implicit rules than with the simple explicit rules.

Since this prediction is unique to the multiple-systems hypothesis, we attempted to conduct an experiment that would produce this pattern of interference. To this end, we reasoned that our secondary task should interfere with the explicit category learning system, but not with the implicit system. As mentioned above, COVIS (Ashby et al., 1998 ) is a neuropsychological theory of category learning that assumes separate explicit and implicit systems. Briefly, the key structures in the COVIS explicit system are the prefrontal cortex, the anterior cingulate, and the head of the caudate nucleus; COVIS assumes that learning in the implicit system is mediated by the tail of the caudate nucleus. Thus, according to COVIS, the ideal secondary task would strongly activate the prefrontal cortex and the anterior cingulate, but not the tail of the caudate nucleus. Recent neuroimaging studies have shown that the anterior cingulate and dorsolateral prefrontal cortex are strongly activated in the Stroop (1935) task (Bench et al., 1993). For this reason, we chose as our secondary task a numerical version of the Stroop task.

Notice that we are assuming that the interference between primary and secondary tasks is a function of the degree to which the two tasks activate the same neural structures. One could also argue that the numerical Stroop task has more task requirements in common with the explicit system assumed in COVIS than with the implicit system. An important example of such a demand is the ability to focus executive attention selectively on a single dimension or component of the stimulus. In the Stroop task, attention must be focused selectively on one aspect of the stimulus (i.e., word color) in the context of a more salient aspect (i.e., word name). With the unidimensional explicit rules used in the present study, selective attention processes are crucial, because one must maintain attention on a single stimulus dimension and ignore the other three dimensions.

Executive selection attention plays a critical role in the COVIS explicit system, but COVIS assumes that the more difficult three-dimensional rules would be learned by the implicit system, which has no formal representation of selective attention. Thus, COVIS assumes that the Stroop task shares more task requirements and overlapping neural structures with explicit category learning than with implicit category learning. Accordingly, the predictions derived from COVIS suggest that the Stroop task should interfere with the learning of explicit rules more than with the learning of implicit rules.

In summary, this experimental design will provide a strong test of the multiple-systems hypothesis. If the concurrent task impairment for explicit rules is less than or equal to the impairment for implicit rules, then we would not be able to reject the single system hypothesis. However, if the concurrent task impairment for the explicit rules is greater than that observed for the implicit rules, then we would have strong evidence for the existence of multiple category learning systems.

\section{METHOD}

\section{Participants}

One hundred thirty undergraduate students at the University of California, Santa Barbara, participated in this experiment in partial fulfillment of a class requirement.

\section{Stimuli and Apparatus}

The stimuli and category structures are described above. Each categorization stimulus subtended a visual angle of $9.5^{\circ}(300 \times$ 300 pixels). The stimuli in the numerical Stroop-type concurrent task were single digits between 2 and 8 that were either 95 pixels in height or 180 pixels in height, subtending $3^{\circ}$ and $5.8^{\circ}$ of visual angle, respectively. The stimuli were presented on a black background using Macintosh PowerPC computers running the Psychophysics Toolbox software (Brainard, 1997; Pelli, 1997) in the Matlab (MathWorks; Sherborn, MA) environment.

\section{Procedure}

In the category learning task, participants classif ied each stimulus into category "A" or category "B" by pressing the appropriate key on the keyboard after the categorization stimulus appeared on the CRT display. The stimulus remained on the screen until a key was pressed. A brief high-pitched tone $(500 \mathrm{~Hz})$ was presented if the response was correct, and a low-pitched tone $(200 \mathrm{~Hz})$ was presented if the response was incorrect. The criterion for learning the category structures was eight consecutive correct responses. After the criterion was met, the participant was instructed by the computer that the categorization rule was about to change. If the participant was not able to achieve the criterion within 200 trials, the trials then terminated and the participant was informed by the computer that the categorization rule was to change.

The experiment was conducted over two sessions separated by a week. This was done to ensure that participants were well practiced with the category learning task and that any interference caused by the concurrent task was not due to learning how to perform the categorization task. Over the course of the two sessions, the partici- 
pants learned four explicit rules and four implicit rules. They never learned the same rule twice. During the first session, the participants learned four rules (two explicit and two implicit) in the following order: explicit, implicit, explicit, implicit. The data from this session were considered practice and were not included in any of the analyses reported below. A week later, the participants were randomly assigned to the concurrent task condition or the control condition. So that we could investigate the temporal dynamics of the concurrent task, the participants learned two sets of two rules. Each set contained an explicit rule and an implicit rule. In order to control for any effects due to the order with which rules were learned, the participants were randomly assigned to one of the two possible rule orders (explicit first or implicit first) for each of the two sets of rules. This yielded four possible rule orders for the second session (E = explicit, I = implicit): EIEI, IEIE, EIIE, IEEI. The specific rule of each type given at any time was randomly determined without replacement for each participant.

In the concurrent task condition (second session), the participants had to perform a numerical analogue of the Stroop task along with learning the four categorization rules. The concurrent task required the participant to remember which of two numbers was physically larger and which was numerically larger. The analogy with the Stroop effect relied on the fact that on $85 \%$ of the trials, the numerically larger number was physically smaller (see, e.g., Algom, Dekel, \& Pansky, 1996; Besner \& Coltheart, 1979). On the remaining $15 \%$ of the trials, the numerically larger number was also physically larger. During a concurrent task trial, the two numbers to be discriminated were presented for $200 \mathrm{msec}$ to the right and to the left of the categorization stimulus, followed by a uniform white mask for another $200 \mathrm{msec}$. The participant was to first respond to the categorization stimulus. Feedback for the categorization task was given immediately following the categorization response. After the categorization feedback was given, the word value or size appeared on the screen, signaling the participant whether to respond with the numerically larger or the physically larger digit, respectively. The participant then responded with the appropriate key as to whether the appropriate digit was on the "right" or the "left." After the concurrent task response, feedback was given in the same manner as for the categorization task. Thus, during the process of making the categorization decision, the participant needed to hold in memory both the numerical value and the physical size of the briefly presented numbers. The participants were instructed to perform the Stroop task perfectly and, "with what was left over," learn the categories. For each participant, any categorization rule in which concurrent task performance was less than $80 \%$ was excluded from further analysis.

\section{RESULTS}

\section{Stroop Task Performance}

Participants performed the concurrent Stroop task with a high degree of accuracy, achieving an overall average of $91.6 \%$ correct. There was no significant difference between Stroop task accuracy on trials when the categorization rule was explicit $(92.1 \%)$ and on those when it was implicit $(91.2 \%)(t=.0001, p>.20)$. There was also no difference between Stroop task accuracy early in the session (91.2\%) and late in the session $(90.7 \%)(t=.006$, $p>.20)$. Finally, there was no significant difference between the control and concurrent task groups with respect to the number of blocks (i.e., category structures) for which the categorization learning criterion (i.e., eight correct responses in a row) was not met within 200 trials $(Z=.95, p>.10)$. Any block for which the categorization learning criterion was not met was excluded from further analysis. In addition, any block for which the Stroop task accuracy was below $80 \%$ was excluded from further analyses, resulting in the exclusion of five blocks of data (two explicit blocks and three implicit blocks all involving different participants).

It is important to note that there was no effect of categorization task type (explicit vs. implicit) on the performance of the Stroop task. This result was expected, given that participants were instructed to perform the Stroop task perfectly and, "with what was left over," perform the categorization task. This equivalence in Stroop task performance between the explicit and implicit categorization groups is crucial for making any claims about the relative effects of the Stroop task on categorization performance.

\section{Category Learning Performance}

A preliminary analysis of the observed data done with Box's test revealed significant differences in the covariance matrices between the control and the concurrent task groups $[F(70,4896)=2.88, p<.001]($ Box, 1950; Winer, 1971). Thus, we proceeded to analyze the data in two ways. First, a $\log _{10}$ transformation of the data was performed to meet the assumptions of the mixed factor analysis of variance (ANOVA). This transformation effectively normalized the covariance matrices across groups $[F(70,4896)=1.292, p>.05]$. A five-way (two within, three between) mixed factor ANOVA was performed on the transformed number of trials taken to reach criterion (eight consecutive correct responses) with the following factors: rule type (within subjects: explicit vs. implicit), session (within subjects: early vs. late), condition (between subjects: control vs. concurrent task), early session rule order (between subjects: explicit first vs. implicit first), late session rule order (between subjects: explicit first vs. implicit first). This analysis revealed a number of important results. First, there was a significant main effect of condition $[F(1,94)=53.31, p<.001]$, showing that the concurrent task group needed more training to learn the category structures than did the control group. Second, there was a marginally significant main effect of early session rule order $[F(1,94)=3.91, p<.051]$, suggesting that if the implicit rule was learned first, more training was required in order to reach criterion for all rules than if the explicit rule was learned first. Third, there was a significant main effect of rule type $[F(1,94)=$ $58.14, p<.001]$, showing that, over all other conditions, explicit rules required less training than did implicit rules. Fourth, there was a significant interaction between rule type and condition $[F(1,94)=4.62, p<.034]$, showing that the concurrent task produced greater interference with explicit rules than with implicit rules. Figure 1 shows the nontransformed data for the early (A) and late (B) session separately.

To provide additional evidence for the crucial rule type $X$ condition interaction, nonparametric tests of interaction (see Bradley, 1968) were performed on the early and late session raw data as well as on the combined early and late 


\section{A) Category Learning Early in Session}

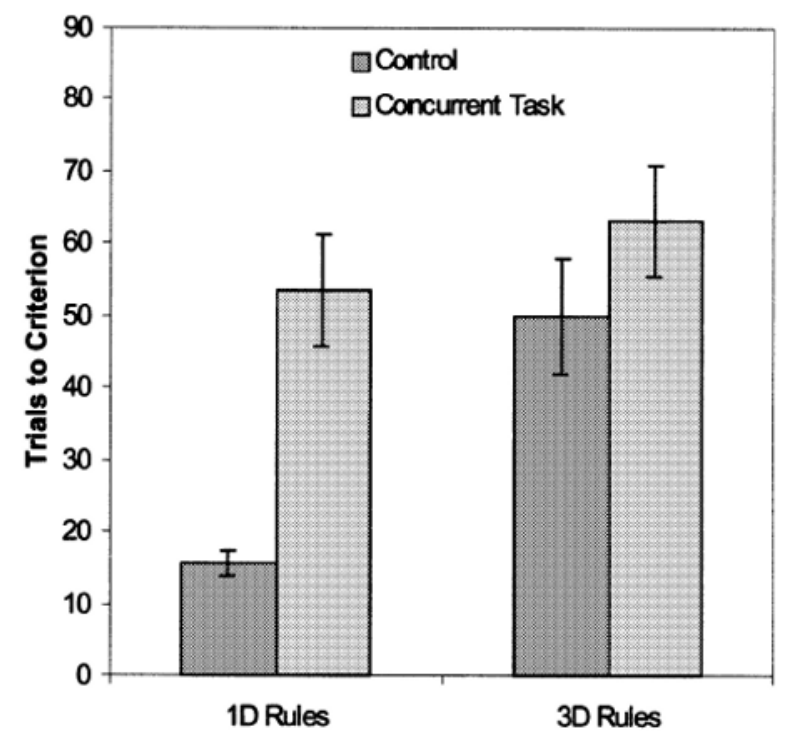

Rule Type

\section{B) Category Learning Late in Session}

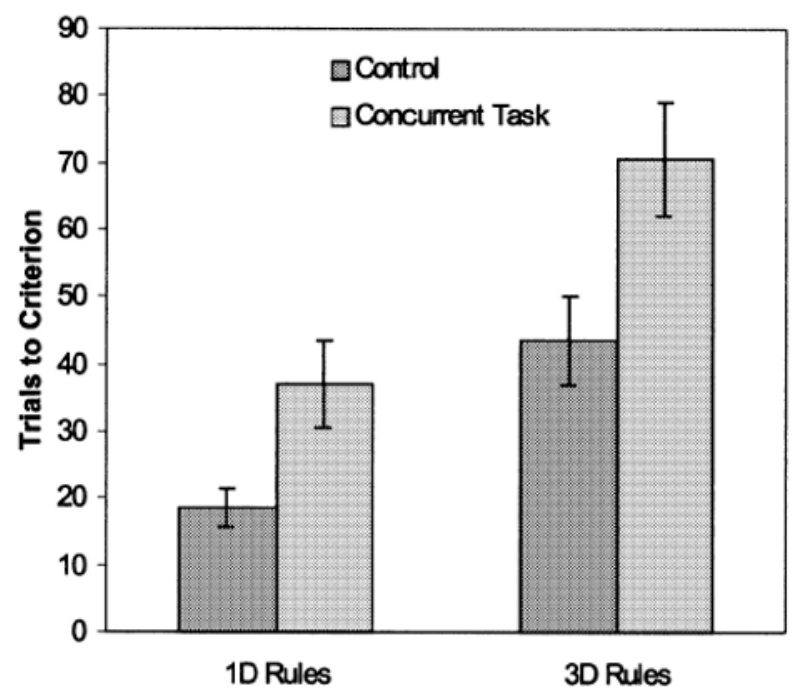

Rule Type

Figure 1. Mean number of trials to criterion for the first two rules learned (A) and for the second two rules learned (B).

session raw data. This analysis revealed a significantinteraction between rule type and condition for the combined data $\left[\chi^{2}(1)=5.58, p<.05\right]$ and for the early session data $\left[\chi^{2}(1)=10.71, p<.01\right]$. For the late session data, the interaction did not reach significance $\left[\chi^{2}(1)=0, p>.05\right]$.

\section{Correlational Analyses}

Since both explicit and implicit rule learning performance were assessed within participants, correlations were carried out to measure the linear relationship between the learning of both rule types. ${ }^{4}$ Partial correlations were computed between the four rules learned on the 2nd day (two explicit, two implicit), controlling for early session rule order, late session rule order, and condition. This analysis showed moderate but significant correlations between the learning of both explicit rules $(r=.24$, $p<.02)$ and both implicit rules $(r=.26, p<.02)$. All correlations between rules of different types were not significant.

\section{DISCUSSION}

These results provide evidence that the explicit and implicit rules used in the present study are learned by qualitatively distinct category learning systems. Relative to control performance, the addition of the concurrent numerical Stroop task produced a profound deficit in the ability of participants to learn simple explicit rules. Single-system models of category learning naturally predict that as the complexity of the categorization rule increases, the deficit caused by the concurrent task should increase because of the increased processing required of more complex rules. However, the present results show the opposite pattern. With the more complex implicit rules that required integrating information from three stimulus dimensions, the interference produced by the addition of the concurrent task was less than that seen with simple unidimensional explicit rules.

Within-subjects correlations between explicit rule learning and implicit rule learning also provide strong support for the multiple-systems hypothesis. If the learning of explicit and implicit rules is mediated by a single system, one would expect to find a strong relationship between the learning of these two types of rules. Specifically, participants who were good at learning one rule type (i.e., implicit) should also be good at learning the other rule type (i.e., explicit). The correlational analyses showed that this was true for rules of the same type, but that the ability to learn explicit rules was unrelated to the ability to learn implicit rules.

One possible weakness in the present results was that the critical rule type $\times$ condition interaction disappears if one considers absolute differences between scores late in the session. ${ }^{5}$ In order to better understand this latesession effect, we carried out pairwise nonparametric comparisons (Wilcoxon signed ranks test) between the early and late session scores for the explicit control, explicit concurrent task, implicit control, and the implicit concurrent task groups. Only the explicit concurrent task group showed a significant improvement across sessions $(Z=2.92, p<.01)$. A natural explanation for why an improvement would be seen in this group is that the concurrent task became easier to perform later in the session and produced less interference. Furthermore, if the explicit and implicit rules were learned by a common system, we would also have expected to see a similar improvement across sessions with the implicit rules. But this improvement in implicit rule learning did not occur. Thus, the late 
A)

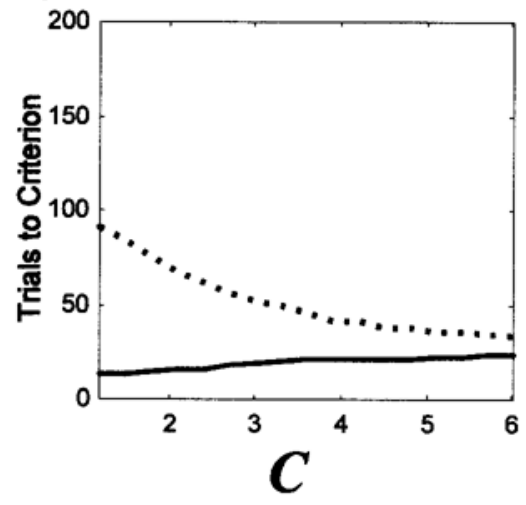

B)

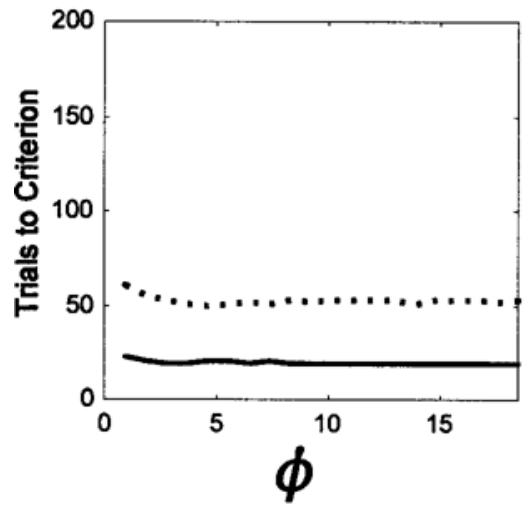

C)

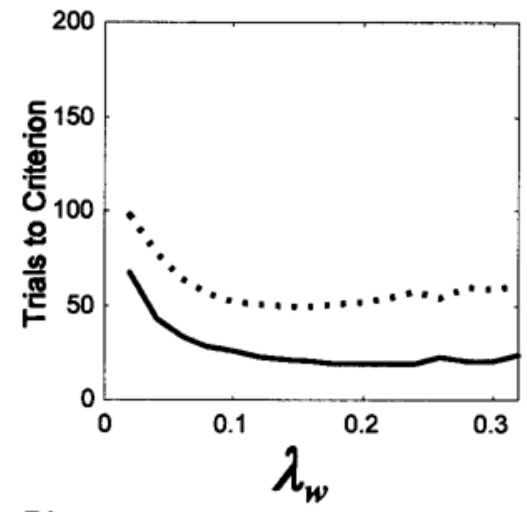

D)

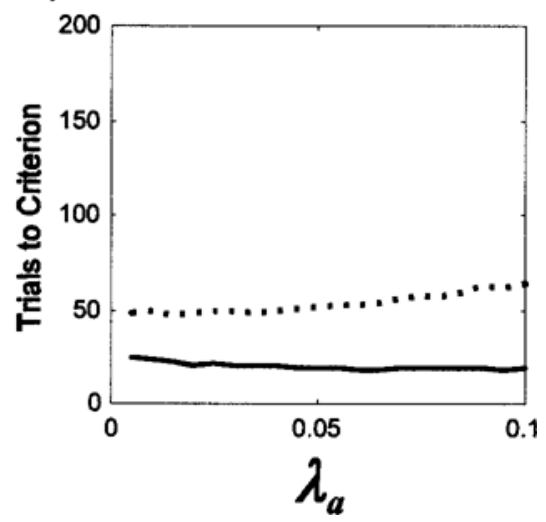

Figure 2. Predictions of ALCOVE for the explicit (solid line) and implicit (broken line) category structures as a function of variation in each of ALCOVE's four parameters.

session effect is actually consistent with the multiplesystems hypothesis.

The present data also have important implications for existing models of category learning. As previously discussed, single-system models naturally predict that the impairment induced by a concurrent task with simple rules should be less than or equal to the impairment with more complex rules. To demonstrate this point more forcefully, we evaluated the predictions of Kruschke's ALCOVE model of category learning (Kruschke, 1992), which is among the most widely known and successful current single-system category learning models.

ALCOVE (attention learning covering map) is an exemplar similarity based connectionist model of category learning. Briefly, it assumes that category decisions are made by computing the similarity of the stimulus to memory representation of all previously seen exemplars (see Kruschke, 1992, for a full description). ALCOVE has four parameters: $c, \phi, \lambda_{w}$, and $\lambda_{a}$. The $c$ parameter is a measure of the overall discriminability of the stimuli, $\phi$ specifies the consistency of responding, and the two learning rates, $\lambda_{w}$ and $\lambda_{a}$, determine how quickly the exemplarcategory associations and attention weights are learned, respectively. The most likely effect of the concurrent task is on the parameter $c$. However, generating precise pre- dictions for the effects of concurrent task interference from ALCOVE is not straightforward, since ALCOVE has not been previously applied to concurrent task experiments. To evaluate the predictions of ALCOVE, we created 1,000 random sequences of trials and recorded how long it took the model to reach a criterion of eight consecutive correct responses for each sequence. The model's predictions were generated by computing the mean trials to criterion over the 1,000 random replications. As with the participant data, we excluded replications for which the model took longer than 200 trials. We then found parameter values that mimicked the pattern of categorization performance observed in the early session control data of the present study. These parameter values were $c=3, \phi=9.24, \lambda_{w}=.2$, and $\lambda_{a}=.05$. With these parameter values, ALCOVE was able to capture accurately the increased difficulty of the implicit structures relative to the explicit structures.

Our concurrent task data contained only two degrees of freedom (i.e., trials to criterion for the explicit and implicit rules, respectively), so an empirical test of the model is possible only under the assumption that the effects of the concurrent task can be modeled by a change in a single parameter. Therefore, we investigated the effects of systematically increasing and decreasing each parameter 


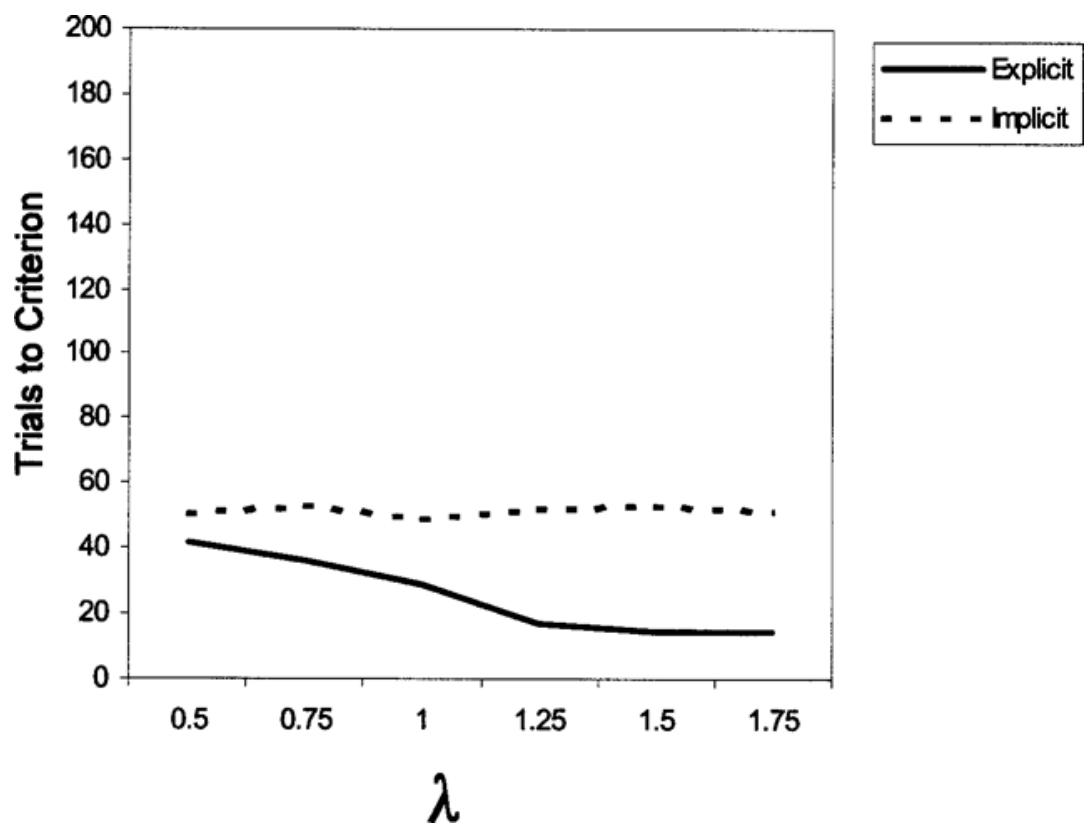

Figure 3. Predictions of COVIS for the explicit (solid line) and implicit (broken line) category structures as a function of variation in the selection parameter.

in turn while the other three parameters were held constant at the same values that best fit the control data. The results of this analysis are shown in Figure 2 for $c$ (Figure 2A), $\phi$ (Figure 2B), $\lambda_{w}$ (Figure 2C), and $\lambda_{a}$ (Figure 2D). Figure 2 shows that for each parameter, any increase in trials to criterion predicted with the explicit category structures is accompanied by a correspondingly large increase in predicted trials to criterion for the implicit category structures. Thus, ALCOVE cannot account for the pattern of performance observed in the present study with any single parameter. Other existing single-system models fail for similar reasons.

Even some dual-process models have difficulty with the present results. For example, consider the RULEX model (rule plus exception; Nosofsky, Palmeri, \& McKinley, 1994), which assumes that in the process of category learning, people apply simple rules and memorize any exceptions to those rules. With explicit rules, RULEX assumes that people only need to find the correct rule, whereas with implicit rules, people need to learn a reasonably effective explicit rule and then memorize any exceptions to that rule. ${ }^{6}$ There are two reasons why RULEX predicts equal or greater concurrent task interference for implicit rules than for explicit rules. First, the processes required in order to learn explicit rules (i.e, rule-based processes) are a proper subset of the processes needed in order to learn implicit rules (i.e., rule-based processes plus memorization). Thus, to the extent that the concurrent task interferes with the learning of unidimensional rules, RULEX predicts a least as much interference with the learning of three-dimensional rules. Second, Nosofsky, Palmeri, and McKinley (1994) explicitly assumed that the memorization process is of limited capacity. Therefore, if the concurrent task reduces the overall capacity available for category learning, the greatest effects should be on the ability of participants to memorize exceptions. For these reasons, RULEX predicts that the concurrent task interference with implicit rules (where exemplar memorization would be necessary) should always be greater than the interference with explicit rules. The opposite pattern was observed in the present data.

On the other hand, the data are consistent with the predictions of COVIS. We used the computational version of COVIS to verify this claim. This is a hybrid neural network consisting of a symbolic learning subsystem (explicit system) that implements logical rules and a pattern classification subsystem (implicit system) that incrementally parses stimulus space into response regions (see Ashby et al., 1998, and Ashby \& Waldron, 1999, for details). As discussed above, COVIS assumes that a common neural locus that is shared by the primary categorization task and the secondary Stroop task is the anterior cingulate. COVIS further assumes that the anterior cingulate is responsible for selecting among competing rules and models this function via a selection parameter $(\lambda)$ that determines how likely a newly selected rule will be implemented. We simulated the present experiment with COVIS in the same manner as for ALCOVE. First we fit the COVIS model to the control data; then we evaluated the predictions of the model for the effects of the concurrent task by varying the $\lambda$ parameter. Figure 3 shows the predicted number of trials to criterion for the explicit (solid line) and implicit (dashed line) tasks as a function of $\lambda$ for the COVIS network. COVIS predicts that as the abil- 
ity to select rules $(\lambda)$ declines, explicit rule learning performance is impaired, whereas implicit rule learning performance is unaffected. Thus, the COVIS network is able to capture the critical aspects of the data, using a single theoretically derived parameter.

\section{CONCLUSIONS}

The present results suggest that performance on the numerical Stroop and explicit categorization tasks depends on a common set of neural mechanisms. COVIS (Ashby et al., 1998) hypothesizes that these structures are part of a frontal striatal thalamic circuit that has also been implicated in selective attention and working memory functions. The present results also suggest that performance on the numerical Stroop and implicit categorization tasks rely on relatively independent processing mechanisms. According to COVIS, the implicit system depends on subcortical structures related to and including the basal ganglia.

The present results also have implications for more general theories of human learning. For example, Hayes and Broadbent (1988) studied explicit and implicit learning under concurrent task conditions. They found concurrent task interference under conditions in which participants could verbalize what they had learned; but in an implicit task that yielded inaccurate verbal reports, a mild concurrent task enhancement was found. The Hayes and Broadbent study was one of the first to demonstrate a performance-based dissociation between learning systems (see Shanks \& St. John, 1994, for a discussion of this issue). However, Green and Shanks (1993) were unable to replicate this important finding. The results reported here, in the context of category learning, provide another performance-based dissociation between learning systems.

It is important to note that in the absence of a double dissociation or a cross-over interaction, the present results do not unequivocally rule out all single-system modelsthat is, it is theoretically possible to construct a singlesystem model that could account for our data. The important point, however, is that, to the best of our knowledge, our results are incompatible with all existing single-system models of category learning (e.g., see Figure 2). At the same time, they are easily and naturally predicted by current multiple systems models (i.e., see Figure 3).

\section{REFERENCES}

Ahn, W. K., \& Medin, D. L. (1992). A two-stage model of category construction. Cognitive Science, 16, 81-121.

Algom, D., Dekel, A., \& Pansky, A. (1996). The perception of number from the separability of the stimulus: The Stroop effect revisited. Memory \& Language, 24, 557-572.

Allen, S. W., \& Brooks, L. R. (1991). Specializing the operation of an explicit rule. Journal of Experimental Psychology: General, 120, 3-19.

Ashby, F. G., Alfonso-Reese, L. A., Turken, A. U., \& Waldron, E. M. (1998). A neuropsychological theory of multiple systems in category learning. Psychological Review, 105, 442-481.

Ashby, F. G., \& Maddox, W. T. (1992). Complex decision rules in categorization: Contrasting novice and experienced performance. Jour- nal of Experimental Psychology: Human Perception \& Performance, 18, 50-71.

Ashby, F. G., Queller, S., \& Berretty, P. M. (1999). On the dominance of unidimensional rules in unsupervised categorization. Perception \& Psychophysics, 61, 1178-1199.

Ashby, F. G., \& WALDRON, E. M. (1999). On the nature of implicit categorization. Psychonomic Bulletin \& Review, 6, 363-378.

Bench, C. J., Frith, C. D., Grasby, P. M., Friston, K. J., Paulesu, E., Frackowiak, R. S. J., \& Dolan, R. J. (1993). Investigations of the functional anatomy of attention using the Stroop test. Neuropsychologia, 31, 907-922.

Besner, D., \& ColtheArt, M. (1979). Ideographic and alphabetic processing in skilled reading of English. Neuropsychologia, 17, 467472.

Box, G. E. P. (1950). Non-normality and tests on variance. Biometrika, 40, 318-335.

Bradley, J. V. (1968). Distribution-free statistical tests. Englewood Cliffs, NJ: Prentice-Hall.

Brainard, D. H. (1997). The Psychophysics Toolbox. Spatial Vision, 10, 443-446.

Brooks, L. (1978). Nonanalytic concept formation and memory for instances. In E. Rosch \& B. B. Lloyd (Eds.), Cognition and categorization (pp. 169-211). Hillsdale, NJ: Erlbaum.

ERICKSON, M. A., \& KruschKe, J. K. (1998). Rules and exemplars in category learning. Journal of Experimental Psychology: General, 127, 107-140.

Estes, W. K. (1986). Array models for category learning. Cognitive Psychology, 18, 500-549.

Green, R. E. A., \& Shanks, D. R. (1993). On the existence of independent explicit and implicit learning systems: An examination of some evidence. Memory \& Language, 21, 304-317.

Hayes, N. A., \& Broadbent, D. E. (1988). Two modes of learning for interactive tasks. Cognition, 28, 249-276.

Hintzman, D. L. (1986). "Schema abstraction" in a multiple-trace memory model. Psychological Review, 93, 411-428.

IMAI, S. \& GARNER, W. R. (1965). Discriminability and preference for attributes in free and constrained classification. Journal of Experimental Psychology, 69, 596-608.

Kendler,H. H., \& Kendler, T. S. (1962). Vertical and horizontal processes in problem solving. Psychological Review, 69, 1-16.

KruschKe, J. K. (1992). ALCOVE: An exemplar-based connectionist model of category learning. Psychological Review, 99, 22-44.

MCKINLEY, S. C., \& NosofSKY, R. M. (1995). Investigations of exemplar and decision bound models in large-size, ill-defined category structures. Journal of Experimental Psychology: Human Perception \& Performance, 21, 128-148.

Medin, D. L., \& Schaffer, M. M. (1978). Context theory of classification learning. Psychological Review, 85, 207-238.

Medin, D. L., \& Schwanenflugel, P. J. (1981). Linear separability in classification learning. Journal of Experimental Psychology: Human Learning \& Memory, 7, 355-368.

Medin, D. L., Wattenmaker, W. D., \& Hampson, S. E. (1987). Family resemblance, conceptual cohesiveness, and category construction. Cognitive Psychology, 19, 242-279.

Mishkin, M., Malamut, B., \& Bachevalier, J. (1984). Memories and habits: Two neural systems. In G. Lynch, J. L. McGaugh, \& N. M. Weinberger (Eds.), Neurobiology of human learning and memory (pp. 65-77). New York: Guilford.

Nosofsky, R. M. (1986). Attention, similarity, and the identificationcategorization relationship. Journal of Experimental Psychology: General, 115, 39-57.

Nosofsky, R. M., Gluck, M. A., Palmeri, T. J., McKinley, S. C., \& Glauthier, P. (1994). Comparing models of rule-based classification learning: A replication and extension of Shepard, Hovland, and Jenkins (1961). Memory \& Cognition, 22, 352-369.

Nosofsky, R. M., \& Johansen, M. K. (2000). Exemplar-based accounts of "multiple-system" phenomena in perceptual categorization. Psychonomic Bulletin \& Review, 7, 375-402.

Nosofsky, R. M., PAlmeri, T. J., \& McKinley, S. C. (1994). Rule-plusexception model of classification learning. Psychological Review, 101, 53-79. 
Pashler, H. (1994). Dual-task interference in simple tasks: Data and theory. Psychological Bulletin, 116, 220-224.

Pelli, D. G. (1997). The VideoToolbox software for visual psychophysics: Transforming numbers into movies. Spatial Vision, 10, 437 442.

SCHACTER, D. L. (1987). Implicit memory: History and current status. Journal of Experimental Psychology: Learning, Memory, \& Cognition, 13, 501-518.

Shanks, D. R., \& ST. John, M. F. (1994). Characteristics of dissociable human learning systems. Behavioral \& Brain Sciences, 17, 367-447.

Shepard, R. N., Hovland, C. I., \& Jenkins H. M. (1961). Learning and memorization of classifications. Psychological Monographs, 75 (13, Whole No. 517).

Smith, E. E., Patalano, A. L., \& Jonides, J. (1998). Alternative strategies of categorization. Cognition, 65, 167-196.

Smith, E. E., Patalano, A. L., Jonides, J., \& Koeppe, R. A. (1996, November). PET evidence for different categorization mechanisms. Paper presented at the 37th Annual Meeting of the Psychonomic Society, Chicago.

Sloman, S. A. (1996). The empirical case for two systems of reasoning. Psychological Bulletin, 119, 3-22.

SQuire, L. R. (1992). Memory and the hippocampus: A synthesis from findings with rats, monkeys, and humans. Psychological Review, 99, 143-145.

STroop, R. J. (1935). Studies of interference in serial verbal reactions. Journal of Experimental Psychology, 18, 643-662.

WATTENMAKER, W. D. (1992). Relational properties and memory-based category construction. Journal of Experimental Psychology: Learning, Memory, \& Cognition, 15, 282-304.

Willingham, D. B., Nissen, M. J., \& Bullemer, P. (1989). On the development of procedural knowledge. Journal of Experimental Psychology: Learning, Memory, \& Cognition, 15, 1047-1060.
WINER, B. J. (1971). Statistical principles in experimental design. New York: McGraw-Hill.

\section{NOTES}

1. In fact, the categories defined by the multidimensional rule were a $45^{\circ}$ rotation of the categories defined by the unidimensional rule.

2. In the Shepard et al. (1961) study, the stimuli varied along three binary-valued dimensions, whereas, the stimuli in the present study varied along four binary-valued dimensions. Thus, the only difference between the category structures investigated by Shepard et al. and the present study is the addition of an irrelevant dimension.

3. Any task requires some explicit processes. Accordingly, COVIS predicts that the concurrent task should produce some interference for the learning of the implicit category structures. However, the interference for the learning of implicit category structures should be less than that observed for the learning of explicit category structures.

4. The correlations were performed on the nontransformed data. The same pattern of results was observed with the $\log _{10}$ transformed data.

5. The analyses based on the log-transformed data, which is a relative scale, did not reveal any effects of Session (early vs. late).

6 . The version of RULEX tested in Nosofsky, Palmeri, and McKinley (1994) assumed that the only rules available to the system were unidimensional rules and two-dimensional rules. Thus, this version of RULEX would assume that subjects would have to memorize exceptions in order to perform optimally in the three-dimensional rule condition used in the present study.

(Manuscript received April 5, 1999; revision accepted for publication April 13, 2000.) 